\title{
Krill oil supplementation reduces the growth of CT-26 orthotopic tumours in Balb/c mice
}

Abilasha Gayani Jayathilake ${ }^{1}$, Elif Kadife ${ }^{1}$, Nyanbol Kuol ${ }^{1}$, Rodney Brain Luwor ${ }^{2}$, Kulmira Nurgali ${ }^{1,3,4+}$ and Xiao Qun Su ${ }^{1 *+}$

\begin{abstract}
Background: We have previously reported that the free fatty acid extract (FFAE) of krill oil (KO) significantly inhibits the proliferation and migration, and induces apoptosis of colorectal cancer (CRC) cells. This study aimed to investigate the in vivo efficacy of various doses of $\mathrm{KO}$ supplementation on the inhibition of CRC tumour growth, molecular markers of proliferation, angiogenesis, apoptosis, the epidermal growth factor receptor (EGFR) and its downstream molecular signalling.
\end{abstract}

Methods: Male Balb/c mice were randomly divided into four groups with five in each group. The control (untreated) group received standard chow diet; and other three groups received $\mathrm{KO}$ supplementation at 5\%, 10\%, and 15\% of their daily dietary intake respectively for three weeks before and after the orthotopic implantation of CT-26 CRC cells in their caecum. The expression of cell proliferation marker Ki-67 and angiogenesis marker CD-31 were assessed by immunohistochemistry. The expression of EGFR, phosphorylated EGFR (pEGFR), protein kinase B (AKT), pAKT, extracellular signal-regulated kinase (ERK1/2), pERK1/2, cleaved caspase-7, cleaved poly (ADP-ribose) polymerase (PARP), and DNA/RNA damage were determined by western blot.

Results: KO supplementation reduced the CRC tumour growth in a dose-dependent manner; with $15 \%$ of KO being the most effective in reduction of tumour weight and volume ( $68.5 \%$ and $68.3 \%$ respectively, $P<0.001)$, inhibition of cell proliferation by $69.9 \%(P<0.001)$ and microvessel density by $72.7 \%(P<0.001)$. The suppressive effects of $\mathrm{KO}$ on EGFR and its downstream signalling, ERK1/2 and AKT, were consistent with our previous in vitro observations. Furthermore, $\mathrm{KO}$ exhibited pro-apoptotic effects on tumour cells as indicated by an increase in the expression of cleaved PARP by 3.9-fold and caspase-7 by 8.9 -fold.

Conclusions: This study has demonstrated that $K O$ supplementation reduces $C R C$ tumour growth by inhibiting cancer cell proliferation and blood vessel formation and inducing apoptosis of tumour cells. These anti-cancer effects are associated with the downregulation of the EGFR signalling pathway and activation of caspase-7, PARP cleavage, and DNA/RNA damage.

Keywords: Krill oil supplementation, Colorectal cancer, CRC tumour growth, Ki-67, CD-31, Caspase-7, PARP, EGFR, ERK $1 / 2$, AKT

*Correspondence: xiao.su@vu.edu.au

${ }^{\dagger}$ Kulmira Nurgali and Xiao Qun Su contributed equally to this work.

${ }^{1}$ Institute for Health and Sport, Victoria University, Melbourne 8001,

Australia

Full list of author information is available at the end of the article

\section{Background}

Colorectal cancer $(\mathrm{CRC})$ is the third most common cancer and the second highest cause of cancer-related deaths in the world [1]. CRC initiates from a gradual progression of genetic and epigenetic alterations in the colonic epithelium. The genetic alterations associated with CRC 
are the mutation in the adenomatous polyposis coli ( $A P C)$ gene and inactivation of the tumour suppressor genes, TP53 (17p) and DCC (18q). Epigenetic alterations including DNA methylation, histone marks, chromatin remodelling and noncoding RNAs (ncRNAs) also play pivotal roles in the development of CRC [2-5]. Genetic and epigenetic aberrations are involved in a complex network and can both predispose to or cause the development of each other [6]. Therefore, understanding epigenetic regulation of gene and/or protein expression can help to develop novel chemotherapy for the treatment of CRC. Multiple factors have been found to be associated with epigenetic regulation and one of such factors is EFGR [7].

It is difficult to detect CRC at the initial stages. The diagnosis is often made at the advanced stages when the tumour has metastasised to other parts of the body. The most common metastasis sites for CRC are the lymph nodes, liver, lungs, peritoneum and the nervous system $[8,9]$. Tumour angiogenesis, resistance to apoptosis and overexpression of EGFR signalling pathways have been identified as factors contributing to tumour growth and metastasis [10-12]. These events, together with increased cell proliferation correlate with the prognosis of CRC [13, 14]. Thus, the development of an effective therapeutic/ adjunct agent that is capable of overcoming these events would be useful to control the CRC growth, given that the currently available chemotherapies are associated with many adverse side effects.

Over the past ten years, several studies have shown that krill oil has therapeutic potential in the treatment of chronic disorders, including inflammation, cardiovascular disease, hyperlipidemia, arthritis, neurological disorders, kidney disease and diabetes [15-17]. Krill oil extracted from Euphausia surperba, a crustacean species found in the Southern Ocean, has been used as an alternative to fish oil due to its high level of long-chain omega- 3 polyunsaturated fatty acids (LC n-3 PUFA) [16, 17]. Furthermore, it has also been reported that the presence of astaxanthin in krill oil increases the stability of LC n-3 PUFA and protects them from oxidation that may lead to better health outcomes than fish oil [18].

Our recent studies showed that the free fatty acid extract (FFAE) of krill oil suppresses the proliferation and induces the apoptosis of human CRC cells through the activation of caspases 3 and 9 and possibly via the mitochondrial death pathway [19, 20]. Zhu et al. [21] and $\mathrm{Su}$ et al. [22] also reported the anti-proliferative effect of krill oil on CRC and osteosarcoma cells. Recently, Zheng and co-authors have observed that krill oil treatment can effectively reduce the growth of various cancer cells such as breast, leukaemia, hepatocellular carcinoma, prostate cancer attributed to the E-configuration structures of krill oil's LC n-3 PUFA, eicosapentaenoic acid (EPA) and docosahexaenoic acid (DHA) [23]. Up to now, only one animal study compared the effect of krill oil treatment with eicosapentaenoic acid monoglyceride (MAGEPA) and EPA ethyl ester (EPA EE) on colorectal tumour growth in nude mice [24]. It was found that krill oil treatment slightly reduced tumour latency and growth compared to control animals.

In this study, we investigated the effects of various doses of krill oil supplementation on CRC tumour growth in orthotopically implanted CT-26 cells in Balb/c mice. The impact of krill oil supplementation on tumour proliferation and angiogenesis was evaluated through the analysis of Ki-67 and CD-31, respectively. Furthermore, the modulatory role of krill oil in vivo on the epidermal growth factor receptor (EGFR) and its downstream molecular signalling, extracellular signal-regulated kinase 1/2 (ERK1/2), and protein kinase B (AKT) were determined. Moreover, the effects of krill oil supplementation on tumour cell apoptosis were determined through the analysis of expression of caspase-7, cleaved poly (ADPribose) polymerase (PARP), and DNA/RNA damage.

\section{Methods \\ CT-26 cells and culture conditions}

The mouse colon cancer cell line, CT-26, was obtained from the American Tissue Culture Collection (ATCC), Manassas, VA, USA (Catalogue No. CRL-2638). Cells were cultured in RPMI1640 medium (Sigma Aldrich, Castle Hill, NSW, Australia) supplemented with foetal calf serum (FCS, 10\%) (Hyclone Quantum Scientific, Clayton South, VIC, Australia), glutamine (10 mM), 4-2-hydroxyethyl-1-piperazineethanesulfonic acid, sodium pyruvate $(10 \mathrm{mM})$, and penicillin $(100 \mathrm{U} / \mathrm{mL}) /$ streptomycin $(100 \mu \mathrm{g} / \mathrm{mL})$ (Sigma Aldrich, Castle Hill, $\mathrm{NSW}$, Australia) at $37{ }^{\circ} \mathrm{C}$ in $5 \% \mathrm{CO}_{2}$ humidified atmosphere. The trypan blue exclusion assay was used to assess the viability of the cancer cells. More than $90 \%$ of viable cells obtained after trypsinization of the monolayer culture were used for CRC induction.

\section{Experimental procedure}

The animal study was approved by the Victoria University Animal Ethics Committee (AEC No. 17/008). The study is reported in accordance with ARRIVE guidelines. The mice were maintained in accordance with the guidelines of the Australian National Health and Medical Research Council Code of Conduct on the care and use of laboratory animals for scientific purposes. Seven-weekold male Balb/c mice $(n=20$, weighted $18-25 \mathrm{~g})$ were obtained from the Animal Resources Centre (Perth, Australia). Animals were housed in OptiMouse cages under pathogen-free conditions with a 12-h light/dark cycle in a well-ventilated room at $22{ }^{\circ} \mathrm{C}$ with free access to food 
and water. Immediately after the arrival, animals were randomised into four groups (untreated group and three treatment groups) with $n=5$ in each group followed by a five-day acclimatisation period. At the end of acclimatisation, Group 1 (untreated/control) continued their standard chow diet (manufactured by Specialty Feeds, Western Australia); Group 2 received a standard chow diet supplemented with 5\% krill oil; Group 3 was given standard chow diet supplemented with $10 \%$ krill oil, and Group 4 received standard chow diet supplemented with 15\% krill oil as shown in Fig. 1. Krill oil, a product from Swisse Wellness Pty Ltd., Victoria, Australia was purchased from a local pharmacy. It contained 13\% EPA, 7.5\% DHA and 0.03\% Astaxanthin. The amount of krill oil provided to individual animals was calculated based on their feed consumption rate (g/day) and adjusted regularly. Oil supplement was mixed with the standard rodent diet by loading into a pre-calibrated hole in the pellet. Diets supplemented with krill oil were prepared fresh and replaced daily to prevent the oxidation of $n-3$ polyunsaturated fatty acids. The dietary supplementation regime followed the study by Bathen et al. [25], with three weeks before and after cancer cell implementation to the animals. At the end of three weeks of pre-treatment, CT-26 cells $\left(1 \times 10^{6}\right.$ cells in $25 \mu \mathrm{L}$ Matrigel, Sigma, Australia) were injected into the caecum of the mice to induce cancer as described before [26]. After a 3-day recovery period, all animals continued their respective dietary treatment for another three weeks. During the study period, food intake was monitored every day and the body weight was measured twice a week. After 21 days of CT-26 cell inoculation or when the tumour size was over $1 \mathrm{~cm}^{3}$, animals were culled by overdosing with pentobarbitone $(100 \mathrm{mg} /$ $\mathrm{kg}$ ) [27]. Kaplan-Meier survival analysis was used to assess the survival rate of animals in each group [28]. The tumours were immediately removed, and their weight and volume were measured. Tumour tissues were snapfrozen in liquid nitrogen and stored at $-80{ }^{\circ} \mathrm{C}$ for later western blotting or fixed with Zamboni's fixative (2\% formaldehyde containing $0.2 \%$ picric acid) overnight at $4{ }^{\circ} \mathrm{C}$ followed by $3 \times 10$ min washes with dimethyl sulfoxide (DMSO) (Sigma, Australia) and $3 \times 10 \mathrm{~min}$ washes with phosphate-buffered saline (PBS). Tissues were frozen in moulds containing 100\% Optimum Cutting Temperature compound (OCT Sakura, Tissue Tek, USA) in liquid nitrogen-cooled isopentane, and stored at $-80{ }^{\circ} \mathrm{C}$ until used for immunohistochemistry analysis.

\section{Immunohistochemistry for Ki-67 and CD-31}

OCT-embedded tumour sections were cut using a cryostat (Leica CM 1950 Biosystems, Germany) at a thickness of $10 \mu \mathrm{m}$. Endogenous peroxidase activity was blocked by incubating slides with $10 \%$ donkey serum at room temperature for $1 \mathrm{~h}$. After $2 \times 10 \mathrm{~min}$ washes with PBS, tumour sections were incubated with primary anti-Ki-67 rabbit monoclonal antibody (1:500 dilution, Abcam, United Kingdom) and anti-CD-31 rat monoclonal antibody (1:500 dilution, Abcam, USA) at $4{ }^{\circ} \mathrm{C}$ overnight. Following $3 \times 10 \mathrm{~min}$ washes with PBS-T (Tween-20 0.05\%), tumour sections were incubated with the secondary antibodies, donkey anti-rabbit Alexa Fluor 488 and donkey anti-rat Alexa Fluor 594 (1:250, Jackson ImmunoResearch Laboratories, USA) at room temperature for $2 \mathrm{~h}$. Slides were then washed $3 \times 10 \mathrm{~min}$ with PBS-T before incubation with 4,6-diamidine-2'-phenylindole dihydrochloride (DAPI, $14 \mathrm{nM}$, Life Technologies, Australia) for $1 \mathrm{~min}$. Finally, slides were washed with PBS-T for $10 \mathrm{~min}$ and cover-slipped using a fluorescent mounting medium (DAKO, Australia). Eight images of each sample at $40 \times$ magnification were taken with the Eclipse Ti confocal laser scanning system (Nikon, Japan). The excitation wavelengths for Alexa Fluor 488 and Alexa Fluor 594 were adjusted to $488 \mathrm{~nm}$ and $559 \mathrm{~nm}$ respectively. All images were then calibrated to standardize for a minimum basal fluorescence and converted to binary. Fluorescence intensity was measured using Image J

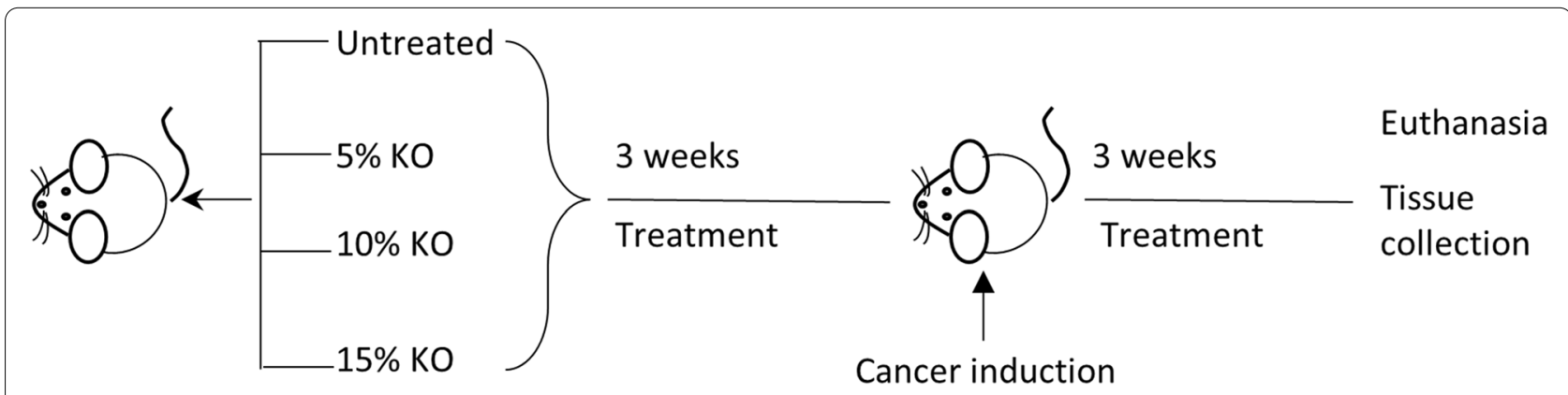

Fig. 1 The schematic overview of the study design investigating the effects of different doses of krill oil supplementation in a mouse model of colorectal cancer 
software (National Institute of Health, USA). All slides were coded, and analysis was performed blindly.

\section{Western blot}

The expressions of pEGFR/EGFR, pERK/ERK 1/2, pAKT/ AKT, active caspase-7, cleaved PARP and DNA/RNA damage proteins were investigated in tumour samples. Frozen tumour samples were homogenised with a polytron homogenizer (Kinematica AG, Switzerland) for $15 \mathrm{~s}$ in ice-cold radioimmunoprecipitation assay (RIPA) buffer (pH 7.4, $150 \mathrm{mM} \mathrm{NaCl}, 0.1 \%$ SDS, 0.5\% sodium deoxycholate, 1\% NP-40 in PBS, Sigma, Australia) containing a protease and phosphatase inhibitors cocktail (Roche Applied Science, USA). The lysis was centrifuged at $12,000 \mathrm{rpm}$ for $20 \mathrm{~min}$ at $4{ }^{\circ} \mathrm{C}$ and supernatants were used for western blot analysis. Pierce bicinchoninic acid (BCA) assay (Thermo Fisher Scientific, Australia) was performed to determine the protein concentration. An equal amount of protein sample $(12 \mu \mathrm{g} / \mathrm{lane})$ was loaded onto Mini-PROTEAN ${ }^{\circledR}$ TGXTM (4-20\%) stain-free precast gel (Bio-Rad, USA) and separated by $10 \%$ sodium dodecyl sulfate-polyacrylamide (SDS-PAGE) gel electrophoresis, then the membrane was blocked with 5\% skim milk in PBST $(0.1 \%$ Tween-20) by incubating at room temperature in a $40 \mathrm{rpm}$ speed shaker for $90 \mathrm{~min}$. The membrane was allowed to react with primary antibodies against pEGFR (1:1000, rabbit, Tyr 1068), EGFR (1:1000, rabbit, monoclonal antibody (mAb), D38B1), pERK 1/2 (1:1000, rabbit, mAB, 9101), ERK 1/2 (1:1000, rabbit, H72), pAKT (1:1000, rabbit, $\mathrm{mAB}, 9271)$, AKT antibody (1:1000, rabbit, 9272) mAB), caspase-7 (1:1000, rabbit, Asp198), PARP (1:1000, rabbit, 9542), (all from Cell Signaling Technology, USA), DNA/RNA damage (1:500, mouse, anti-8-OHdG mAB, ab62623 [15A3], Abcam, USA), and glyceraldehyde-3-phosphate dehydrogenase (GAPDH) (1:2000 dilution, rabbit, Santa Cruz Biotechnology, USA) used as the loading control and incubated overnight at $4{ }^{\circ} \mathrm{C}$. Following $3 \times$ washes in PBS-T $(0.1 \%$ Tween-20) the membrane was incubated with secondary antibodies, goat anti-rabbit IgG H\&L horseradish peroxidase (HRP) (Abcam, ab6721, USA) and horse anti-mouse IgG (1:10,000, Cell Signaling Technology, MA, USA) at room temperature for $1 \mathrm{~h}$. Again, the membrane was washed three times in PBS-T (0.1\% Tween-20). The protein detection was carried out using enhancing chemiluminescence reagents (Clarity ${ }^{\mathrm{TM}}$ Western ECL Substrate, Bio-Rad, USA). Chemiluminescence signals were captured using the FUSION FX System (USA). The expression level of each protein was quantified using Fusion Capt Advance FX7 software. The results were verified through at least three individual experiments.

\section{Statistical analysis}

All data were analysed using SPSS 22 software (IBM, USA). Mixed model ANOVA was used to determine the significance between treatments. The significance of repeated measures at different time points was analysed using one-way ANOVA. Post-hoc analysis was conducted using the Tukey HSD test for multiple comparisons. $P<0.05$ was considered significant. The Kaplan-Meier survival analysis was performed to assess the survival rate of animals in each group using the formula: Survival Rate $=$ (number of subjects living at the start - number of subjects died)/ number of subjects living at the start. One-way ANOVA was performed to compare the survival rate of animals in different experimental groups. The results were expressed as mean $\pm S D$ in texts and mean \pm SEM in figures.

\section{Results \\ Effects of different doses of krill oil supplementation on animal survival rate and tumour growth}

Animals received a 3-week dietary supplementation of different doses of krill oil in both pre- and post-cancer induction periods (Fig. 1). There were no significant changes in the body weights following krill oil treatment compared to the untreated group (Fig. 2A). The survival rate after the induction of CRC was improved significantly in animals received $10 \%$ and $15 \%$ of krill oil supplementation compared to the untreated animals, as shown in Fig. 2B.

The total weight of the caecum with tumours excised from animals treated with different doses of krill oil compared to the caecum from the untreated group is presented in Fig. 3A. The average caecum weight of the untreated animals was $2.8 \pm 0.2 \mathrm{~g}$, while for the groups fed with $5 \%$ and $10 \%$ krill oil, caecum weights were $2.4 \pm 0.1 \mathrm{~g}$ and $2.1 \pm 0.2 \mathrm{~g}$, respectively. The reduction was $16.1 \%$ and $24.1 \%$, respectively, although not statistically significant. Animals treated with $15 \%$ of krill oil showed the lowest caecum weight with an average of $1.5 \pm 0.1 \mathrm{~g}$, indicating a significant reduction by $55.4 \%$ compared to the untreated group $(P<0.01)$ as shown in Fig. 3B. Moreover, untreated animals fed with a standard chow diet exhibited an aggressive tumour growth in the wall of the caecum, and tumour metastases were observed in various organs/tissues including the colon, small intestine, liver, and diaphragm. Mice fed with $5 \%$ of krill oil supplementation also showed more intensive tumour growth in the caecum and metastases in other sites similar to untreated animals. In contrast, all animals treated with $10 \%$ or $15 \%$ of krill oil had a relatively reduced number and size of tumours in the wall of the caecum compared to the untreated animals, with the most remarkable 

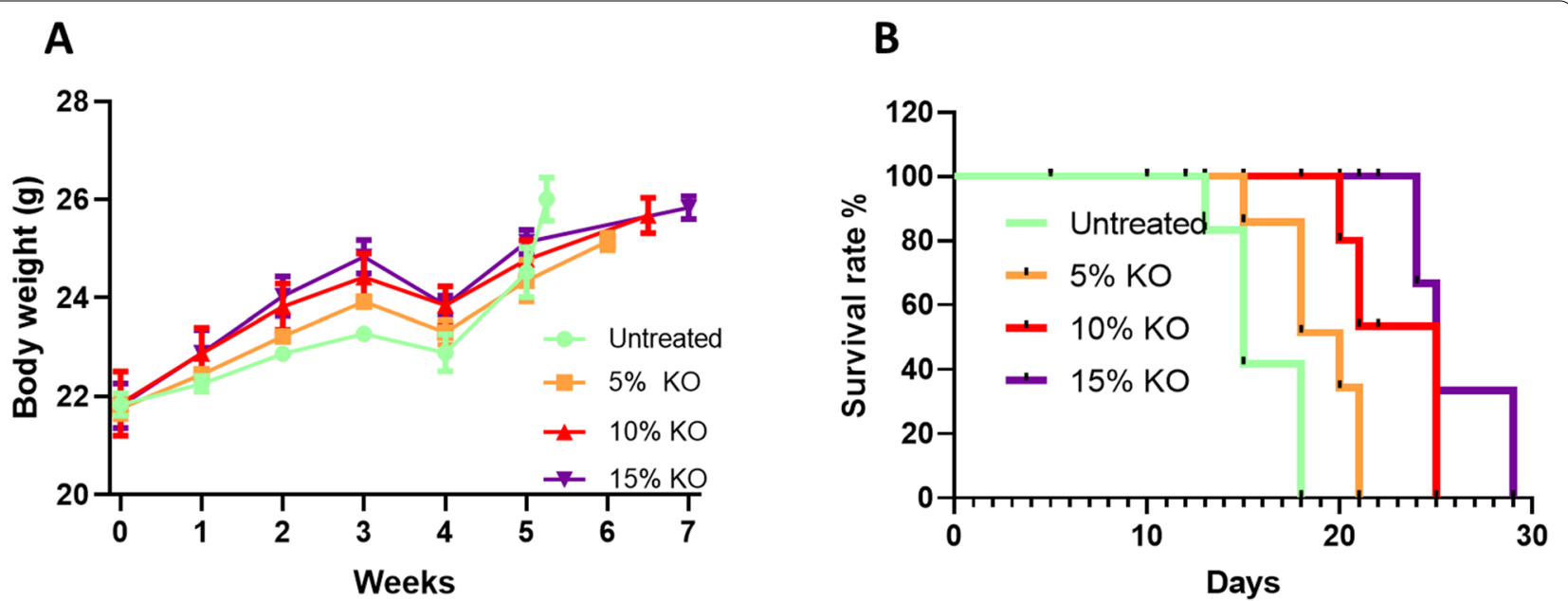

Fig. 2 Effects of krill oil supplementation on body weight and animal survival. A Changes in the mouse body weight following krill oil treatment compared to the untreated group. B Kaplan-Meier survival plot representing the percentage of survival of mice treated with krill oil compared to the untreated group

changes in the group treated with $15 \%$ of krill oil. In both $10 \%$ and $15 \%$ of krill oil-treated groups, only 1 out of 5 mice had metastases to other organs.

The total mean weight and volume of tumours from the untreated group were $2.8 \pm 0.3 \mathrm{~g}$ and $2659.7 \mathrm{~mm}^{3}$, respectively. No differences in the tumour weight and volume were found between the group treated with $5 \%$ of krill oil $\left(2.3 \pm 0.2 \mathrm{~g}\right.$ and $2283,1 \mathrm{~mm}^{3}$, respectively) and the untreated group (Figs. $3 \mathrm{C}$ and D). The average tumour weight in these animals was reduced by $17.4 \%$ and the volume by $19.4 \%$ compared to the untreated animals, although the difference was not statistically significant $(P>0.05)$. For mice fed with the diet supplemented with $10 \%$ of krill oil, the average tumour weight and volume were $1.4 \pm 0.2 \mathrm{~g}$ and $1371.6 \mathrm{~mm}^{3}$, respectively. These indicate a reduction in tumour weight by $48.6 \%$ and tumour volume by $51.6 \%$ compared to the untreated group $(P<0.01$ for both). The average weight and volume of tumours in animals fed the diet supplemented with $15 \%$ of krill oil were $0.9 \pm 0.1 \mathrm{~g}$ and $898.7 \mathrm{~mm}^{3}$, respectively, indicating a significant reduction in tumour weight by $68.5 \%$ and volume by $68.3 \%$ compared to untreated animals $(P<0.001$ for both) (Figs. $3 \mathrm{C}$ and $\mathrm{D})$.

\section{Effects of different doses of krill oil supplementation on tumour cell proliferation}

Tumour cell proliferation was analysed immunohistochemically using a proliferation marker Ki-67 (Fig. 4A). The animals fed the diets supplemented with 5\% and 10\% of krill oil showed a reduction of the number of proliferative cells in the tumour by $22.5 \pm 5.2 \%$ and $42.0 \pm 2.6 \%$ $(P<0.01$ and $P<0.001)$, respectively, compared to the untreated group. The animals fed with $15 \%$ of krill oil showed the lowest number of Ki-67 positive cells in the tumour, with a significant reduction by $69.9 \pm 2.0 \%$ $(P<0.001)$ compared to the untreated group. This is significantly lower compared to animals fed with $10 \%$ of krill oil by $25.2 \%(P<0.05)$ and animals fed with $5 \%$ of krill oil by $61.1 \%(P<0.001)$ (Figs. $4 \mathrm{~B}$ and $C$ ).

\section{Effects of different doses of krill oil supplementation on tumour vascularisation}

The tumour vascularisation was determined using the endothelial cell marker CD-31 (Fig. 5A). The animals received diets with krill oil supplementations at concentrations of $5 \%, 10 \%$, and $15 \%$ have shown a significantly lower expression of CD-31 by $49.3 \%(P<0.01), 64.3 \%$ $(P<0.001)$, and $72.7 \%(P<0.001)$ respectively compared to untreated animals. Moreover, CD-31 expression in tumours from mice treated with $15 \%$ of krill oil was significantly lower compared to mice treated with $5 \%$ of krill oil $(P<0.01)$. This indicates that krill oil supplementation inhibits the blood vessel formation in colorectal tumours in a dose-dependent manner (Fig. 5B).

\section{Expression of pEGFR/EGFR, pERK/ERK $1 / 2$ and pAKT/AKT following krill oil supplementation}

The expressions of pEGFR, EGFR, pAKT, AKT, pERK1/2, and ERK1/2 protein levels were investigated in tumours from the animals fed with $15 \%$ of krill oil (Fig. 6A). The western blot results revealed that the expression levels of EGFR, AKT, and total ERK1/2 were not changed in the animals fed with $15 \%$ of krill oil compared to the untreated animals. However, the expression of pEGFR, 
A

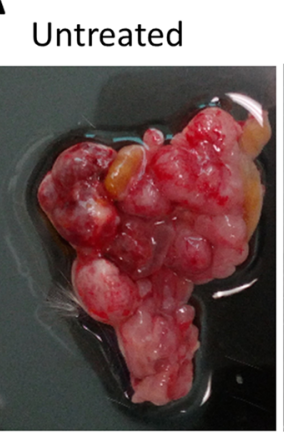

$5 \% \mathrm{KO}$

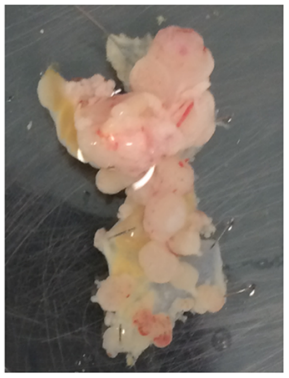

$10 \% \mathrm{KO}$

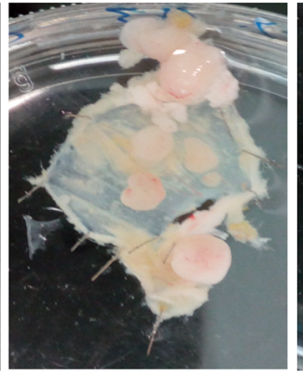

$15 \%$ KO

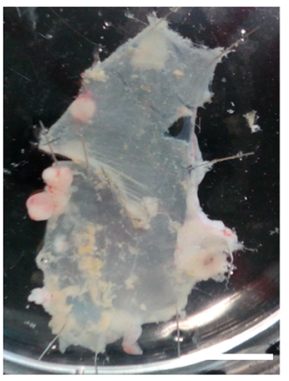

B

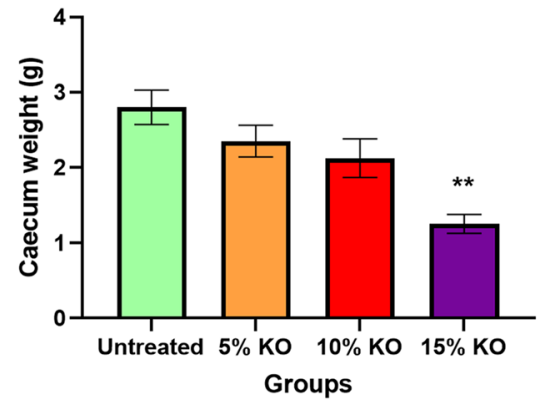

C

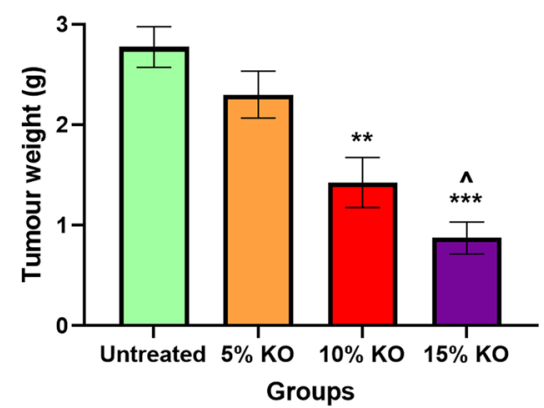

D

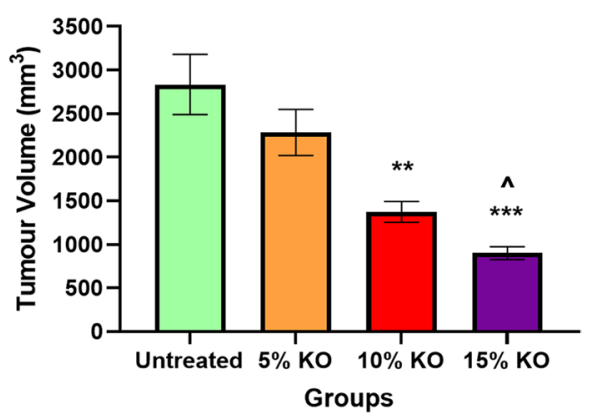

Fig. 3 Effects of krill oil supplementation on the caecum and tumour weight and volume in Balb/c mice. A Images of mouse caecum implanted with CT-26 cells following treatments with different doses of krill oil compared to the untreated group. B The average weight of the caecum with tumours from animals treated with krill oil compared to untreated animals. C The mean tumour weight in mice following treatment with different doses of krill oil compared to the untreated group. D The mean tumour volume in mice treated with different doses of krill oil compared to untreated animals. $N=5$ mice per group. The results are expressed as mean $\pm S E M,{ }^{*} P<0.05,{ }^{* *} P<0.01$, and ${ }^{* * *} P<0.001$ compared to the untreated group; $\wedge P<0.05$ compared to the $5 \%$ krill oil-treated group

pAKT, and pERK1/2were significantly lower in the animals fed with $15 \%$ of krill oil with $0.45,0.54$ and 0.46 folds reduction, respectively $(P<0.001$ for all $)$ compared to the untreated animals (Fig. 6B).

\section{Expression of cleaved caspase-7, PARP, and DNA/RNA damage following krill oil supplementation}

The results of western blot analysis of tumours showed that krill oil supplementation activates caspase-7 in a dose-dependent manner (Fig. 7A). The animals fed diets supplemented with $10 \%$ and $15 \%$ of krill oil showed an increase in the expression of cleaved caspase- 7 by 9.6 and 9.9 folds, respectively, compared to the untreated animals $(P<0.001)$. The difference between the group fed with $5 \%$ of krill oil and the untreated group was not statistically significant (Fig. 7B).

Furthermore, the animals fed with $10 \%$ and $15 \%$ of krill oil showed a dose-dependent change in the expression of PARP protein with an increase of 3.6 and 3.9 folds, respectively, compared to the untreated animals $(P<0.001$ for both) (Fig. 7C). Moreover, the increase in the level of DNA/RNA damage was found following the 

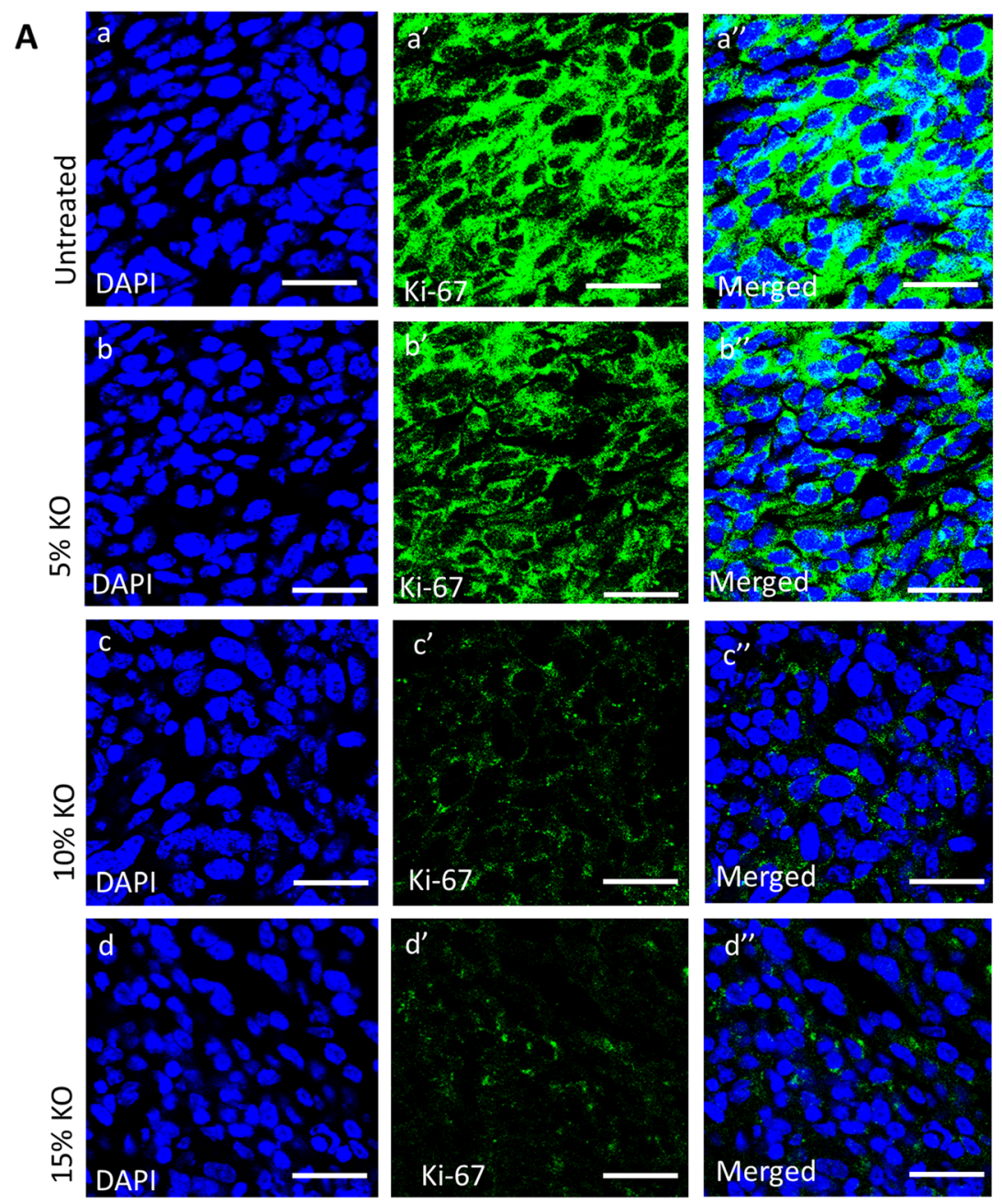

B

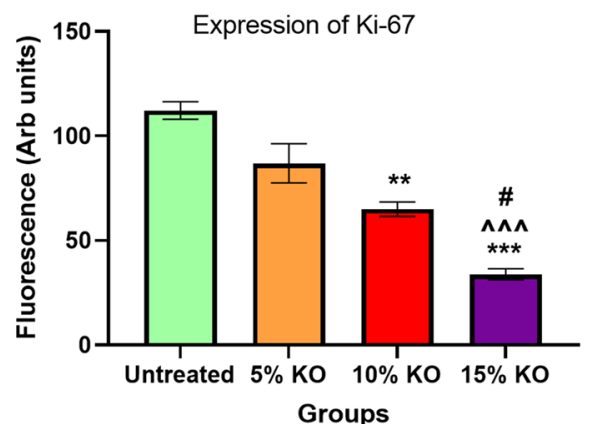

C

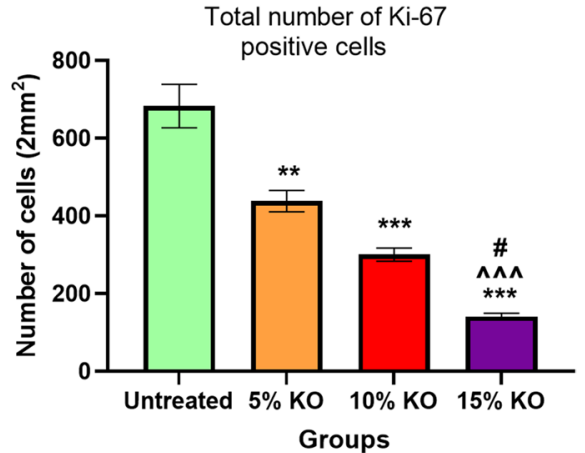

Fig. 4 Expression of Ki-67 following treatments with different doses of krill oil. A The expression of Ki-67 in tumours following in vivo treatments with different doses of krill oil compared to the untreated group was determined using a monoclonal antibody for Ki-67. B The fluorescence intensity of Ki-67 expression in tumours following treatments with different doses of krill oil compared to the untreated group. C Quantification of Ki-67-positive cells based on 8 images per preparation at $20 \times$ magnification within a total area of $2 \mathrm{~mm}^{2}$. The thickness of tumour cross-sections $=10 \mu \mathrm{M} . N=5$ mice per group. The results are expressed as mean $\pm S E M$, ${ }^{* *} P<0.01$, and ${ }^{* * *} P<0.001$ compared to the untreated group; $\wedge \wedge \wedge P<0.001$ compared to $5 \%$ krill oil-treated group; ${ }^{\#} P<0.05$ compared to $10 \%$ krill oil-treated group 

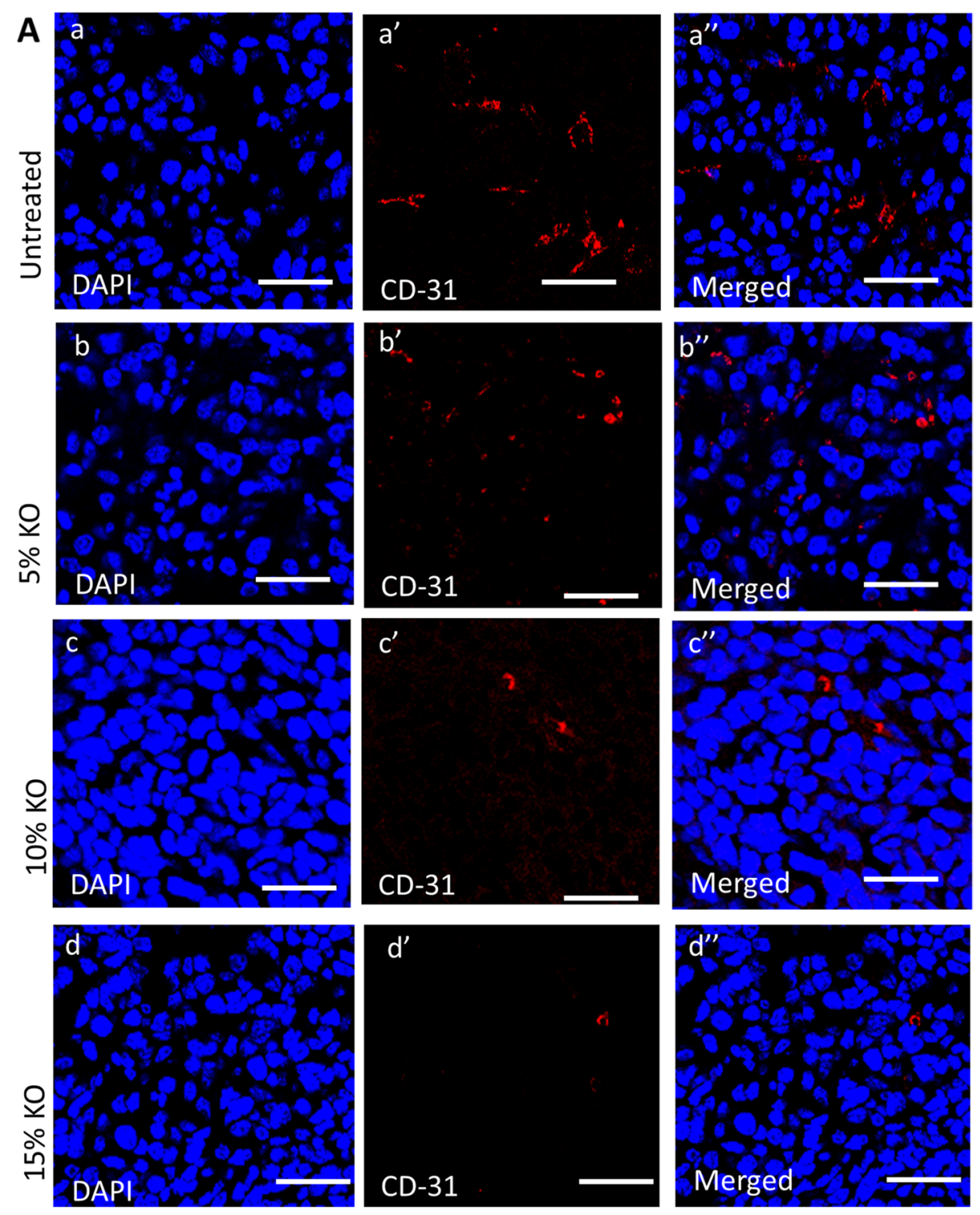

B

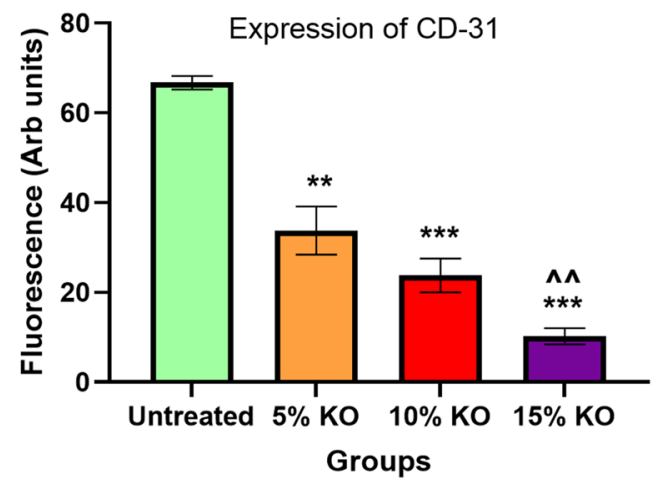

Fig. 5 Expression of CD-31 following treatments with different doses of krill oil. (A) The expression of CD-31 in tumours following in vivo treatments with different doses of krill oil compared to the untreated group was determined using a monoclonal antibody for CD-31. (B) The fluorescent intensity of CD-31 expression in tumours following treatments with different doses of krill oil compared to the untreated group. Thickens of tumour cross-sections $=10 \mu \mathrm{M} . \mathrm{N}=5$ mice per group, the results are expressed as mean $\pm \mathrm{SEM}{ }^{* *} P<0.01$, and ${ }^{* *} P<0.001$ compared to the untreated group; $\wedge \wedge P<0.01$ compared to the $5 \%$ krill oil-treated group 
A

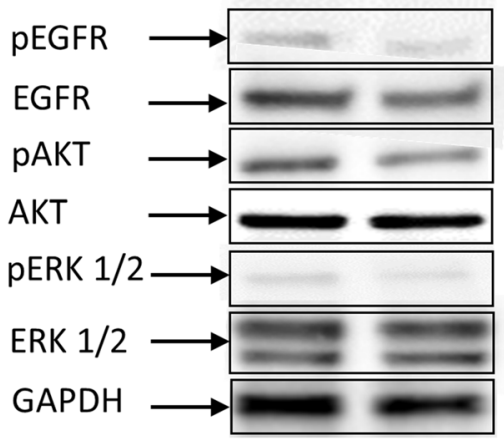

B

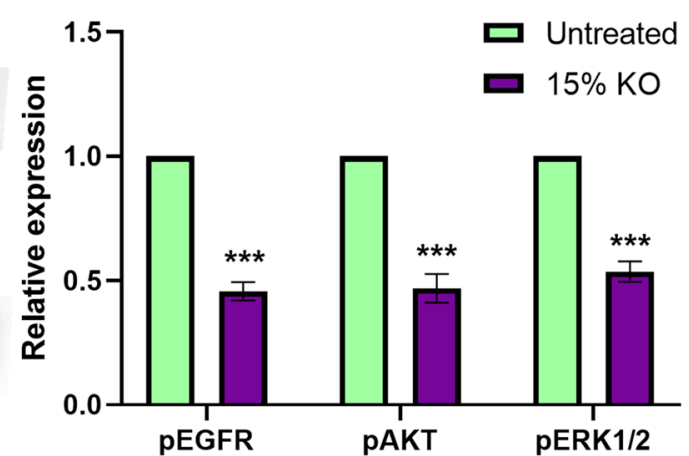

Fig. 6 Expression of pEGFR, EGFR, pAKT, AKT pERK 1/2, and ERK 1/2 following treatments with 15\% of krill oil. The expression of pEGFR, EGFR, pAKT, AKT, pERK $1 / 2$, and ERK $1 / 2$ was analysed by western blotting in tumours following treatment with a $15 \%$ dietary krill oil supplementation compared to the untreated group $\mathbf{A}$. The relative levels of pEGFR, pAKT, and pERK1/2 expression in tumour tissues after treatment with $15 \%$ krill oil compared to tumour tissues from untreated Balb/c mice $\mathbf{B}$. $N=5$ mice per group. The results are expressed as mean $\pm S E M,{ }^{* * *} P<0.001$ compared to the untreated group

A

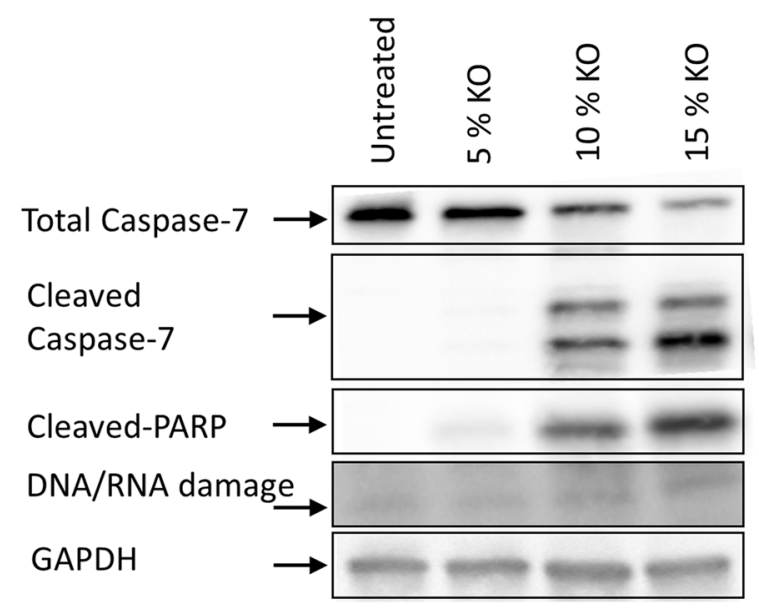

B

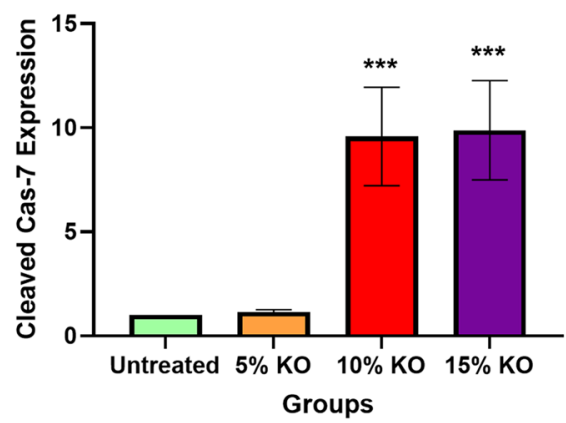

C

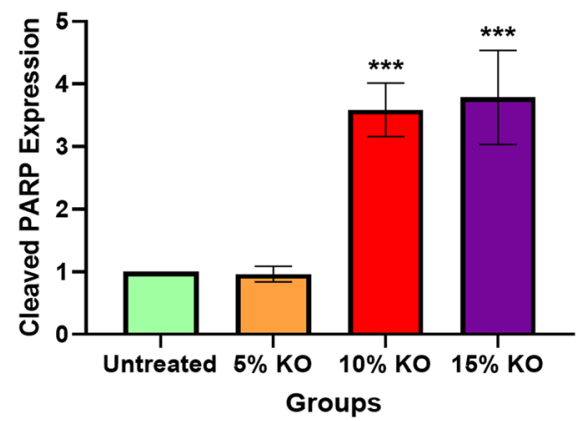

Fig. 7 Expression of caspase-7, cleaved caspase-7, cleaved PARP, and DNA/RNA damage following treatments with different doses of krill oil. The expression of Caspase-7, cleaved caspase-7, cleaved PARP, and DNA/RNA damage was analysed by western blotting in tumours following treatment with different doses of krill oil compared to the untreated group $\mathbf{A}$. The relative level of cleaved caspase-7 expression in tumours following treatment with different doses of krill oil compared to tumour tissues from untreated Balb/c mice $\mathbf{B}$. The relative level of cleaved PARP expression in tumours after treatment with different doses of krill oil compared to tumour tissues from untreated Balb/c mice $\mathbf{C}$. N= 5 mice per group. The results are expressed as mean \pm SEM, ${ }^{* * *} P<0.001$ compared to the untreated group 
treatments with $10 \%$ and $15 \%$ of krill oil by $31.8 \%$ and $34.2 \%$, respectively, compared to untreated animals (Fig. 7A).

\section{Discussion}

In this study, we have demonstrated that dietary krill oil supplementation inhibits the growth of CRC tumour in Balb/c mice. The main findings of the study include: 1 ) krill oil supplementation reduces tumour volume, invasion, and metastasis in a dose-dependent manner within a range of $10-15 \%$ of daily feed intake, and $15 \%$ of krill oil being the most effective dose; 2) krill oil supplementation correspondingly reduces tumour cell proliferation and angiogenesis; 3) krill oil downregulates the expression of pEGFR, and its downstream pERK1/2 and pAKT signalling pathways in colorectal tumours in the same fashion as reported in our previous in vitro study using the free fatty acid extract of krill oil; 4) krill oil supplementation upregulates the expression of cleaved caspase-7, cleaved PARP, and DNA/RNA damage in tumours.

The hallmarks of cancer include uncontrolled cell proliferation, inhibition of apoptosis and induction of angiogenesis, resulting in the growth and progression of the solid tumour [29]. Disruption of the tightly regulated EGFR pathway was found to be involved in many cellular processes, including proliferation, growth, and survival of neoplastic cells [30,31]. Many studies have observed the activation of various carcinogenic events that induce cancer development and progression. Therefore, targeting a single event is not sufficient for tumour control [29, 32]. Accordingly, most cancer treatments aim to control multiple events rather than a single factor in order to suppress CRC progression [33, 34], such as inhibiting cell survival, signalling pathways, and angiogenesis, as well as inducing cellular apoptosis $[35,36]$. Hence, in the present study, we investigated the anti-tumour potential of krill oil in a pre-clinical animal model of CRC, by examining its impact on various molecular markers/signalling pathways that are related to cell proliferation, invasion, angiogenesis, and apoptosis.

Krill oil contains a higher level of LC n-3 PUFA, EPA and DHA, and the anti-cancer properties of these LC n-3 PUFA have been reported in both in vitro $[37,38]$ and in vivo studies [25, 39]. For example, Morin et al. [24] have evaluated the anti-cancer effect of EPA monoglyceride (MAG-EPA) and krill oil treatment on HCT-116 cells and also in a mouse model of HCT-116 xenografts. They found that MAG-EPA at the dose of $618 \mathrm{mg} / \mathrm{kg} / \mathrm{day}$ reduced cell proliferation and tumour size by up to $75 \%$, but no significant reduction was observed after krill oil treatment in vivo. In their study, the administrated dose of krill oil was $618 \mathrm{mg} / \mathrm{kg} /$ day (less than $5 \%$ ). The current study also showed that treatment with $5 \%$ of krill oil does not result in positive effects against CRC. The study by Latham et al. [40] observed that fish oil at the dose of $80 \mathrm{~g} / \mathrm{kg}$ (EPA 18.7\% and DHA $8.0 \%$ ) consumption significantly reduces the incidents of aberrant crypt foci (ACF) in the distal colon following administration of 1,2-dimethylhydrazine (DMH) to induce cancer in Wistar rats. Rosa et al. [41] also reported that $4 \%$ of fish oil-supplemented diet given to Wister rats resulted in a significant reduction of ACF formation in the proximal colon after receiving DMH injection.

Our study showed that $15 \%$ of krill oil supplementation reduces tumour weight and volume by $68.5 \%$ and $68.3 \%$, respectively, compared to untreated animals. The inhibitory effects of krill oil on tumour growth are most likely associated with the suppression of proliferation and induction of apoptosis of cancer cells. This was supported by our results demonstrating that krill oil supplementation induces apoptosis and downregulation of Ki-67 in the tumours. The Ki-67 is a biomarker for the proliferation of tumour cells and is a regulatory protein associated with the nuclear cell cycle. Its expression can be detected during the interface in the nucleus of tumour epithelial cells [42, 43]. In addition, Ki-67 is involved in the entire active phases of the cell cycle, G1, S, G2, and mitosis, but is absent in the resting phase of the cell cycle (G0 phase) [44]. Therefore, Ki-67 is an excellent marker for determining the growth fraction of tumours [45]. These in vivo results are consistent with our previous findings demonstrating that the FFAE of krill oil exhibited a significant anti-proliferative effect on human osteosarcoma and CRC cells [20, 22].

The formation of a vascular network is crucial for increased cell proliferation and metastatic spread of tumour as this network supplies oxygen, nutrients, and eliminates waste products that are essential for the progression of cancer $[46,47]$. Furthermore, tumour-associated angiogenesis is believed to be dependent on the production of pro-angiogenic growth factors by tumour cells. These factors enhance tumour angiogenesis to promote tumour growth [48]. Studies also showed that suppression of angiogenesis obstructs tumour growth and progression [49]. Therefore, therapeutic approaches that can inhibit angiogenesis would help to control tumorigenesis $[50,51]$. In the present study, we found that higher doses of krill oil (10-15\%) significantly inhibit blood vessel formation in colorectal tumours, evident by the reduced expression of platelet endothelial cell adhesion molecule CD-31. CD-31 is highly expressed on the surface of all vascular cells. It is a well-defined marker for identifying angiogenesis and also well-established for monitoring blood vessel density in malignant tissues $[52,53]$. On the contrary, a higher expression of this biomarker was observed in the untreated group with highly 
aggressive tumours. There was no significant change in the expression of CD-31 following treatment with the low dose of krill oil (5\%) compared to the untreated group. The effect of krill oil on CD-31 may be attributed to the presence of LC n-3 PUFA in this marine oil. Previously, Hawcroft et al. [54] have demonstrated low doses of EPA-FFA at 2.5 to $5 \%$ ww- 1 did not decrease the density of CD31-positive microvessels in CRC tumour tissues which correlated with the lack of CRC cell apoptosis in metastatic liver tumours in Balb/c mice. These findings are consistent with the results of our study, demonstrating that only higher doses of krill oil supplementation (10-15\%) were effective in reducing CD-31 expression, but not the lower doses of krill oil (5\%). A decreased density of CD-31- positive microvessels in Met-1 tumours from FVB female mice was observed following 17 days of treatment with epoxydocosapentaenoic acids (EDPs), lipid mediators produced from DHA by cytochrome P450 epoxygenases [55].

In this study, we observed the downregulation of phosphorylated EGFR and its ERK1/2 and AKT downstream signalling pathways with no alternations of their total protein levels in tumours following krill oil supplementation in vivo. These results are consistent with our previous findings that the free fatty acid extract of krill oil inhibits the expression of EGFR and its downstream signalling pathway, leading to CRC cancer cell death [56]. EGFR signalling pathways are responsible for various biological processes, including cell growth, proliferation, and survival [57]. The overexpression of EGFR and its ERK1/2 and AKT downstream signalling pathways is common in cancer including CRC. The EGFR mutation results in dysregulation of several biological processes, including cell proliferation, apoptosis, and survival $[58,59]$. Furthermore, previous studies have observed that activation of the EGFR pathway also increases the synthesis of angiogenic molecules in different types of tumour cells [60-63]. Taken together, it suggests that, the anti-cancer properties of krill oil observed in the present study may be associated with the downregulation of pEGFR, pERK1/2, and pAKT signalling, leading to the inhibition of tumour cell proliferation, angiogenesis and apoptosis.

Furthermore, the anti-proliferative and pro-apoptotic effects of krill oil may be attributed to its role in the activation of caspase-7, hence inducing cellular apoptosis through the cleavage of PARP and finally induced DNA/ RNA damage. The extrinsic and intrinsic apoptotic signalling cascades are the two commonly distinguished independent apoptotic pathways [64]. The interaction of death receptor ligands causes the activation of the extrinsic apoptotic pathway through activation of caspase- 8 and caspase-10. On the other hand, the intrinsic apoptotic pathway is triggered by the change of mitochondrial membrane potential and release of cytochrome $c$ in the cytosol and this causes the formation of the apoptosome complex to combine with caspase-9. Once activated, caspase-9 leads to the activation of the executioner caspase- 3 and -7 to induce apoptosis $[65,66]$. In our previous in vitro study, we have reported the role of FFAE of krill oil in the activation of caspase- 9 and -3 . The results of the present in vivo study demonstrate almost tenfold increases in the expression of cleaved caspase- 7 in tumours from mice with $10 \%$ and $15 \%$ krill oil supplementation. It has been reported that there is a functional link between caspase-7 and PARP cleavage involved in both inflammation and apoptotic processes [67]. While the current study has demonstrated that krill oil supplementation reduces CRC tumour growth via the activation of caspase-7, which results in PARP cleavage to induce DNA damage, Lamkanfi et al. [67] have reported that the caspase-7-induced apoptosis depends on the cell type and stimulus type. Further studies are required to elucidate whether krill oil supplementation affects inflammatory pathways leading to caspase-7 activation.

Collectively, the present study shows that krill oil supplementation inhibits colorectal tumours growth in Balb/c mice. This effect is most likely related to the anti-proliferative, anti-angiogenic, and pro-apoptotic properties of krill oil. In addition, krill oil supplementation did not show any side effects, such as vomiting, diarrhoea, or constipation throughout the study period. There were also no adverse effects on food intake and body weight. This is consistent with the previous studies on SD rats and Wistar rats. Robertson et al. [68] have found that 13-week supplementation with krill oil in Wistar rats has not caused adverse toxicological effects on animals. The studies by Zhu et al. [21] and Ramsvik et al. [69] observed that treatment with krill oil can control the serum lipid level without any side effects.

The limitation of this pilot study is that only a small number of animals were used for each treatment. An increase in the number of animals in each group would improve the statistical power. Furthermore, the study did not include treatments with isolated active components of krill oil, including EPA, DHA, and astaxanthin, therefore it is not possible to identify the specific roles of these components in relation to the anti-cancer properties of krill oil. In addition, the activity and RT-PCR of Caspase 7 were not measured in this study. Future investigation on these aspects will help to further understand the molecular mechanisms underlying the anti-tumour effects of krill oil.

\section{Conclusion}

This pilot animal study has demonstrated that krill oil supplementation reduces CRC tumour growth in a dose-dependent manner (5\% to15\%). The anti-tumour 
effects of krill oil may be associated with the suppression of CRC cell proliferation through the downregulation of EGFR signalling pathways in the same manner as in our previous in vitro study as well as the activation of caspase-7 to induce apoptosis and inhibition of tumour angiogenesis. The results of this pilot study could be used as a basis for larger animal studies or human clinical trials to further explore the anti-cancer potential of krill oil.

\begin{abstract}
Abbreviations
AKT: Protein kinase B; ATCC: American tissue culture collection; CD-31: Platelet endothelial cell adhesion molecule; CRC: Colorectal cancer; DAPI: 4,6-Diamidino-2-phenylindole; DHA: Docosahexaenoic acid; EGFR: Epidermal growth factor receptor; EPA: Eicosapentaenoic acid; ERK: Extracellular signal regulated kinase; FCS: Foetal calf serum; FFA: Free fatty acids; FFAE of KO: Free fatty acids extract of krill oil; GAPDH: Glyceraldehydes-3-phosphate de-hydrogenase; HEPES: 4-(2-Hydroxyethyl)-1-piperazineethanesulfonic acid; LC n-3 PUFA: Long chain $\mathrm{n}-3$ polyunsaturated fatty acids; MAPK: Mitogen-activated protein kinase; OCT: Optimal cutting temperature compound; pAKT: Phosphorylated AKT; PBS: Phosphate-buffered saline; PBS-T: Phosphate-buffered saline + Tween 20; pEGFR: Phosphorylated EGFR; pERK: phosphorylated ERK; PARP: Cleaved poly (ADP-ribose) polymerase; PUFA: Polyunsaturated fatty acids; RIPA: Radioimmunoprecipitation assay buffer; RPM: Revolutions per minute; SD: Standard deviation; SDS: Sodium dodecyl sulphate; SEM: Standard error of mean; TP53: Tumour protein p53.
\end{abstract}

\section{Supplementary Information}

The online version contains supplementary material available at https://doi. org/10.1186/s12906-022-03521-4.

Additional file 1. Supplementary Figure 6 A. Supplementary Figure 7.

\section{Acknowledgements}

Not applicable.

\section{Authors' contributions}

$\mathrm{XS}, \mathrm{KN}$, and RL designed the study. AJ collected and analysed the data and drafted the manuscript. EK assisted with data collection, analysis, and manuscript editing. NK assisted with the animal study. XS, KN, and RL are responsible for data interpretation and contributed to the critical revision of the manuscript. All authors read and approved the final manuscript.

\section{Funding}

Open Access funding enabled and organized by CAUL and its Member Institutions. This study was supported by Victoria University postgraduate research scholarship for AJ.

\section{Availability of data and materials}

The datasets from the present study are available from the corresponding author upon request.

\section{Declarations}

\section{Competing interests}

The authors declare no competing interests.

\section{Ethics approval and consent to participate}

The animal study was approved by the Victoria University Animal Ethics Committee (ethics number AEC 17-008). All methods were performed in accordance with the guidelines of the Australian National Health and Medical Research Council Code of Conduct on the care and use of laboratory animals for scientific purposes.

\section{Consent for publication}

Not applicable.

\section{Competing of interests}

The authors declare that they have no competing interests.

\section{Author details}

${ }^{1}$ Institute for Health and Sport, Victoria University, Melbourne 8001, Australia. ${ }^{2}$ Department of Surgery, The Royal Melbourne Hospital, The University of Melbourne, Parkville, Australia. ${ }^{3}$ Department of Medicine, Western Health, The University of Melbourne, Melbourne, Australia. ${ }^{4}$ Regenerative Medicine and Stem Cells Program, Australian Institute for Musculoskeletal Sciences (AIMSS), Melbourne, Australia.

Received: 6 August 2021 Accepted: 25 January 2022

Published online: 04 February 2022

\section{References}

1. Bray F, Ferlay J, Soerjomataram I, Siegel RL, Torre LA, Jemal A. Global cancer statistics 2018: GLOBOCAN estimates of incidence and mortality worldwide for 36 cancers in 185 countries. CA Cancer J Clin. 2018;68(6):394-424.

2. Fearon ER, Vogelstein B. A genetic model for colorectal tumorigenesis. Cell. 1990;61(5):759-67.

3. Hong SN. Genetic and epigenetic alterations of colorectal cancer. Intest Res. 2018;16(3):327-37.

4. Rodrigues NR, Rowan A, Smith ME, Kerr IB, Bodmer WF, Gannon JV, et al. p53 mutations in colorectal cancer. Proc Natl Acad Sci U S A. 1990;87(19):7555-9.

5. Patnaik S, Anupriya. Drugs targeting epigenetic modifications and plausible therapeutic strategies against colorectal cancer. Front pharmacol. 2019;6(10):588.

6. Vaiopoulos AG, Athanasoula KC, Papavassiliou AG. Epigenetic modifications in colorectal cancer: Molecular insights and therapeutic challenges. Biochim Biophys Acta. 2014;1842(7):971-80.

7. Amirkhah R, Naderi-Meshkin H, Shah JS, Dunne PD, Schmitz U. The Intricate Interplay between Epigenetic Events, Alternative Splicing and Noncoding RNA Deregulation in Colorectal Cancer. Cells. 2019;8(8):929.

8. Hugen N, van de Velde CJ, de Wilt JH, Nagtegaal ID. Metastatic pattern in colorectal cancer is strongly influenced by histological subtype. Ann Oncol. 2014;25(3):651-7.

9. Riihimaki M, Hemminki A, Sundquist J, Hemminki K. Patterns of metastasis in colon and rectal cancer. Sci Rep. 2016:6:29765.

10. Battaglin F, Puccini A, Intini R, Schirripa M, Ferro A, Bergamo F, et al. The role of tumor angiogenesis as a therapeutic target in colorectal cancer. Expert Rev Anticancer Ther. 2018;18(3):251-66.

11 Giordano G, Remo A, Porras A, Pancione M. Immune Resistance and EGFR Antagonists in Colorectal Cancer. Cancers. 2019;11(8):1089.

12. Watson AJ. Apoptosis and colorectal cancer. Gut. 2004;53(11):1701-9.

13. Gerl R, Vaux DL. Apoptosis in the development and treatment of cancer. Carcinogenesis. 2005;26(2):263-70.

14. Igney $\mathrm{FH}, \mathrm{Krammer} \mathrm{PH}$. Death and anti-death: tumour resistance to apoptosis. Nat Rev Cancer. 2002;2(4):277-88.

15. Burri L, Johnsen L. Krill products: an overview of animal studies. Nutrients. 2015;7(5):3300-21.

16. Tou JC, Jaczynski J, Chen YC. Krill for human consumption: nutritional value and potential health benefits. Nutr Rev. 2007;65(2):63-77.

17. Winther B, Hoem N, Berge K, Reubsaet L. Elucidation of phosphatidylcholine composition in krill oil extracted from Euphausia superba. Lipids. 2011:46(1):25-36.

18. Berge RK, Ramsvik MS, Bohov P, Svardal A, Nordrehaug JE, Rostrup E, et al. Krill oil reduces plasma triacylglycerol level and improves related lipoprotein particle concentration, fatty acid composition and redox status in healthy young adults - a pilot study. Lipids Health Dis. 2015;14:163.

19. Jayathilake AG, Kadife E, Luwor RB, Nurgali K, Su XQ. Krill oil extract suppresses the proliferation of colorectal cancer cells through activation of caspase 3/9. Nutr Metab (Lond). 2019;16:53.

20. Jayathilake AG, Senior PV, Su XQ. Krill oil extract suppresses cell growth and induces apoptosis of human colorectal cancer cells. BMC Complement Altern Med. 2016;16(1):328. 
21. Zhu JJ, Shi JH, Qian WB, Cai ZZ, Li D. Effects of krill oil on serum lipids of hyperlipidemic rats and human SW480 cells. Lipids Health Dis. 2008;7:30.

22. Su X, Tanalgo P, Bustos M, Dass CR. The Effect of Krill Oil and n-3 Polyunsaturated Fatty Acids on Human Osteosarcoma Cell Proliferation and Migration. Curr Drug Targets. 2018;19(5):479-86.

23. Zheng W, Wang X, Cao W, Yang B, Mu Y, Dong Y, et al. E-configuration structures of EPA and DHA derived from Euphausia superba and their significant inhibitive effects on growth of human cancer cell lines in vitro. Prostaglandins Leukot Essent Fatty Acids. 2017;117:47-53.

24 Morin C, Rodriguez E, Blier PU, Fortin S. Potential Application of Eicosapentaenoic Acid Monoacylglyceride in the Management of Colorectal Cancer. Mar Drugs. 2017:15(9):283.

25. Bathen TF, Holmgren K, Lundemo AG, Hjelstuen MH, Krokan HE, Gribbestad IS, et al. Omega-3 fatty acids suppress growth of SW620 human colon cancer xenografts in nude mice. Anticancer Res. 2008;28(6A):3717-23.

26. Miller S, Senior PV, Prakash M, Apostolopoulos V, Sakkal S, Nurgali K. Leukocyte populations and IL-6 in the tumor microenvironment of an orthotopic colorectal cancer model. Acta Biochim Biophys Sin. 2016;48(4):334-41.

27. Reimer JN, Schuster CJ, Knight CG, Pang DSJ, Leung VSY. Intraperitoneal injection of sodium pentobarbital has the potential to elicit pain in adult rats (Rattus norvegicus). PloS One. 2020;15(9):e0238123.

28. Goel MK, Khanna P, Kishore J. Understanding survival analysis: KaplanMeier estimate. Int J Ayurveda Res. 2010;1(4):274-8.

29. Hanahan D, Weinberg RA. Hallmarks of cancer: the next generation. Cell. 2011;144(5):646-74.

30 Krasinskas AM. EGFR Signaling in Colorectal Carcinoma. Patholog Res Int. 2011;2011:932932.

31. Moustacchi E. Molecular mechanisms of carcinogenesis: the role of systems of DNA repair. Bull Acad Natl Med. 1998;182(1):33-46 discussion 7.

32. Teimouri H, Kochugaeva MP, Kolomeisky AB. Elucidating the correlations between cancer initiation times and lifetime cancer risks. Sci Rep. 2019:9(1):18940

33. Kanthan R, Senger J-L, Kanthan SC. Molecular events in primary and metastatic colorectal carcinoma: a review. Patholo Res Int. 2012:2012:597497.

34. Xie Y-H, Chen Y-X, Fang J-Y. Comprehensive review of targeted therapy for colorectal cancer. Signal Transduct Target Ther. 2020;5(1):22.

35. An W, Lai H, Zhang Y, Liu M, Lin X, Cao S. Apoptotic Pathway as the Therapeutic Target for Anticancer Traditional Chinese Medicines. Front Pharmacol. 2019;10:758

36. Khan M, Maryam A, Qazi Jl, Ma T. Targeting Apoptosis and Multiple Signaling Pathways with Icariside II in Cancer Cells. Int J Biol Sci. 2015;11(9):1100-12.

37. Weng WH, Leung WH, Pang YJ, Kuo LW, Hsu HH. EPA significantly improves anti-EGFR targeted therapy by regulating miR-378 expression in colorectal cancer. Oncol Lett. 2018;16(5):6188-94.

38. Zhang C, Yu H, Ni X, Shen S, Das UN. Growth inhibitory effect of polyunsaturated fatty acids (PUFAs) on colon cancer cells via their growth inhibitory metabolites and fatty acid composition changes. PloS One. 2015;10(4):e0123256.

39. Zhang K, Hu Z, Qi H, Shi Z, Chang Y, Yao Q, et al. G-protein-coupled receptors mediate omega-3 PUFAs-inhibited colorectal cancer by activating the Hippo pathway. Oncotarget. 2016;7(36):58315-30.

40. Latham P, Lund EK, Johnson IT. Dietary n-3 PUFA increases the apoptotic response to 1,2-dimethylhydrazine, reduces mitosis and suppresses the induction of carcinogenesis in the rat colon. Carcinogenesis. 1999;20(4):645-50.

41. Rosa DD, Lourenco FC, da Fonseca AC, de Sales RL, Ribeiro SM, Neves $\mathrm{CA}$, et al. Fish oil improves the lipid profile and reduces inflammatory cytokines in Wistar rats with precancerous colon lesions. Nutr Cancer. 2012;64(4):569-79.

42. Richardsen E, Andersen S, Al-Saad S, Rakaee M, Nordby Y, Pedersen MI, et al. Evaluation of the proliferation marker Ki-67 in a large prostatectomy cohort. PLoS One. 2017;12(11):e0186852

43. Scholzen T, Endl E, Wohlenberg C, van der Sar S, Cowell IG, Gerdes J, et al. The Ki-67 protein interacts with members of the heterochromatin protein 1 (HP1) family: a potential role in the regulation of higher-order chromatin structure. J Pathol. 2002;196(2):135-44.
44. Gerdes J, Lemke H, Baisch H, Wacker HH, Schwab U, Stein H. Cell cycle analysis of a cell proliferation-associated human nuclear antigen defined by the monoclonal antibody Ki-67. J Immunol. 1984;133(4):1710-5.

45. Yerushalmi R, Woods R, Ravdin PM, Hayes MM, Gelmon KA. Ki67 in breast cancer: prognostic and predictive potential. Lancet Oncol. 2010;11(2):174-83.

46. Nishida N, Yano H, Nishida T, Kamura T, Kojiro M. Angiogenesis in cancer. Vasc Health Risk Manag. 2006;2(3):213-9.

47. Saman H, Raza SS, Uddin S, Rasul K. Inducing angiogenesis, a key step in cancer vascularization, and treatment approaches. Cancers. 2020;12(5):1172.

48. Huang Z, Bao SD. Roles of main pro- and anti-angiogenic factors in tumor angiogenesis. World J Gastroenterol. 2004;10(4):463-70.

49. El-Kenawi AE, El-Remessy AB. Angiogenesis inhibitors in cancer therapy: mechanistic perspective on classification and treatment rationales. $\mathrm{Br} J$ Pharmacol. 2013;170(4):712-29.

50. Nussenbaum F, Herman IM. Tumor Angiogenesis: Insights and Innovations. J Oncol. 2010;2010:132641.

51. Wang Z, Dabrosin C, Yin X, Fuster MM, Arreola A, Rathmell WK, et al. Broad targeting of angiogenesis for cancer prevention and therapy. Semin Cancer Biol. 2015;35 Suppl(Suppl):S224-43.

52. Gumina RJ, Kirschbaum NE, Rao PN, vanTuinen P, Newman PJ. The human PECAM1 gene maps to 17q23. Genomics. 1996;34(2):229-32.

53. Schluter A, Weller P, Kanaan O, Nel I, Heusgen L, Hoing B, et al. CD31 and VEGF are prognostic biomarkers in early-stage, but not in late-stage, laryngeal squamous cell carcinoma. BMC Cancer. 2018;18(1):272.

54. Hawcroft G, Volpato M, Marston G, Ingram N, Perry SL, Cockbain AJ, et al. The omega-3 polyunsaturated fatty acid eicosapentaenoic acid inhibits mouse MC-26 colorectal cancer cell liver metastasis via inhibition of PGE2-dependent cell motility. Br J Pharmacol. 2012;166(5):1724-37.

55. Zhang G, Panigrahy D, Mahakian LM, Yang J, Liu J-Y, Stephen Lee $\mathrm{KS}$, et al. Epoxy metabolites of docosahexaenoic acid (DHA) inhibit angiogenesis, tumor growth, and metastasis. Proc Natl Acad Sci. 2013;110(16):6530-5.

56. Jayathilake AG, Veale MF, Luwor RB, Nurgali K, Su XQ. Krill oil extract inhibits the migration of human colorectal cancer cells and down-regulates EGFR signalling and PD-L1 expression. BMC Complement Med Ther. 2020;20(1):372.

57. Janmaat ML, Giaccone G. The epidermal growth factor receptor pathway and its inhibition as anticancer therapy. Drugs of Today. 2003;39 Suppl C:61-80.

58. Cohen RB. Epidermal growth factor receptor as a therapeutic target in colorectal cancer. Clin Colorectal Cancer. 2003;2(4):246-51.

59. Sasaki T, Hiroki K, Yamashita Y. The role of epidermal growth factor receptor in cancer metastasis and microenvironment. BioMed Res Int. 2013;2013:546318.

60. Akagi M, Kawaguchi M, Liu W, McCarty MF, Takeda A, Fan F, et al. Induction of neuropilin-1 and vascular endothelial growth factor by epidermal growth factor in human gastric cancer cells. Br J Cancer. 2003:88(5):796-802.

61. Goldman CK, Kim J, Wong WL, King V, BrockT, Gillespie GY. Epidermal growth factor stimulates vascular endothelial growth factor production by human malignant glioma cells: a model of glioblastoma multiforme pathophysiology. Mol Biol Cell. 1993:4(1):121-33.

62. Minder P, Zajac E, Quigley JP, Deryugina EI. EGFR regulates the development and microarchitecture of intratumoral angiogenic vasculature capable of sustaining cancer cell intravasation. Neoplasia. 2015:17(8):634-49.

63. van Cruijsen H, Giaccone G, Hoekman K. Epidermal growth factor receptor and angiogenesis: Opportunities for combined anticancer strategies. Int J Cancer. 2005;117(6):883-8.

64. Fulda S, Debatin KM. Extrinsic versus intrinsic apoptosis pathways in anticancer chemotherapy. Oncogene. 2006;25(34):4798-811.

65. Brentnall M, Rodriguez-Menocal L, De Guevara RL, Cepero E, Boise LH. Caspase-9, caspase-3 and caspase-7 have distinct roles during intrinsic apoptosis. BMC Cell Biol. 2013;14(1):32.

66. D'Eliseo D, Di Rocco G, Loria R, Soddu S, Santoni A, Velotti F. Epitelialto-mesenchimal transition and invasion are upmodulated by tumorexpressed granzyme $B$ and inhibited by docosahexaenoic acid in human colorectal cancer cells. J of Exp Clin Cancer Res. 2016;35:24.

67. Lamkanfi M, Kanneganti TD. Caspase-7: a protease involved in apoptosis and inflammation. Int J Biochem Cell Biol. 2010:42(1):21-4. 
68. Robertson B, Burri L, Berge K. Genotoxicity test and subchronic toxicity study with Superba krill oil in rats. Toxicol Rep. 2014;1:764-76.

69. Ramsvik MS, Bjorndal B, Bruheim I, Bohov P, Berge RK. A PhospholipidProtein Complex from Krill with Antioxidative and Immunomodulating Properties Reduced Plasma Triacylglycerol and Hepatic Lipogenesis in Rats. Mar Drugs. 2015;13(7):4375-97.

\section{Publisher's Note}

Springer Nature remains neutral with regard to jurisdictional claims in published maps and institutional affiliations.

- fast, convenient online submission

- thorough peer review by experienced researchers in your field

- rapid publication on acceptance

- support for research data, including large and complex data types

- gold Open Access which fosters wider collaboration and increased citations

- maximum visibility for your research: over $100 \mathrm{M}$ website views per year

At $\mathrm{BMC}$, research is always in progress.

Learn more biomedcentral.com/submissions 